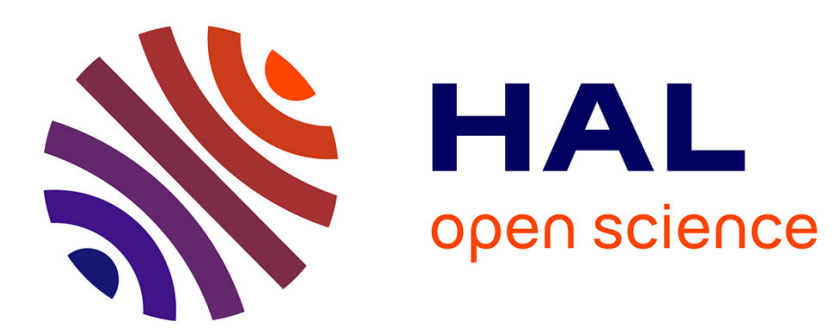

\title{
Quelques exemples d'utilisation de la microscopie électronique à balayage pour l'étude morphologique de matériaux vitreux
}

\author{
M. Murat, A. Negro
}

\section{- To cite this version:}

M. Murat, A. Negro. Quelques exemples d'utilisation de la microscopie électronique à balayage pour l'étude morphologique de matériaux vitreux. Revue de Physique Appliquée, 1974, 9 (2), pp.403-418. 10.1051/rphysap:0197400902040300 . jpa-00243795

\section{HAL Id: jpa-00243795 https://hal.science/jpa-00243795}

Submitted on 1 Jan 1974

HAL is a multi-disciplinary open access archive for the deposit and dissemination of scientific research documents, whether they are published or not. The documents may come from teaching and research institutions in France or abroad, or from public or private research centers.
L'archive ouverte pluridisciplinaire HAL, est destinée au dépôt et à la diffusion de documents scientifiques de niveau recherche, publiés ou non, émanant des établissements d'enseignement et de recherche français ou étrangers, des laboratoires publics ou privés. 


\title{
QUELQUES EXEMPLES D'UTILISATION \\ DE LA MICROSCOPIE ÉLECTRONIQUE A BALAYAGE POUR L'ÉTUDE MORPHOLOGIQUE DE MATÉRIAUX VITREUX
}

\author{
M. MURAT \\ Université Claude-Bernard, Lyon I, \\ Laboratoire de Chimie Appliquée et de Génie Chimique, ERA CNRS no 300, France
}

et A. NEGRO

Politecnico, di Torino, Istituto di Chimica Generale e Applicata, Italie

\begin{abstract}
Résumé. - L'étude porte sur deux catégories de solides :
- des matériaux silicatés vitreux ou partiellement vitrifiés, utilisés dans l'industrie des liants hydrauliques (clinkers de ciment portland, cendres volantes de centrales thermiques, laitiers de hauts fourneaux) ;

- des verres de cordiérite $2 \mathrm{MgO}, 2 \mathrm{Al}_{2} \mathrm{O}_{3}, 5 \mathrm{SiO}_{2}$ traités thermiquement à l'état pur ou en présence de quantités croissantes d'alumine, de zircone, d'oxyde de titane ou d'oxyde de chrome $\mathrm{Cr}_{2} \mathrm{O}_{3}$, substances ajoutées intentionnellement aux fins de contrôle des processus de recristallisation. - La microscopie électronique à balayage (appareil MEB 07 de CAMECA) permet de différencier très nettement, en fonction de leur origine, la morphologie et la texture des premiers matériaux cités, ces facteurs jouant un rôle très important dans le comportement thermique ou la réactivité de ces solides vis-à-vis de l'eau (hydraulicité). Dans le cas des verres de cordiérite, la technique expérimentale permet de suivre les phénomènes de recristallisation thermique, lesquels sont très sensiblement influencés selon la nature ou la teneur de l'oxyde utilisé comme agent de germination, et d'obtenir des informations très intéressantes sur la texture interne des céramiques vitreuses.
\end{abstract}

\begin{abstract}
The study deals with two series of solids :
- glassy or partly glassy materials used in the industry of hydraulic binders (portland cement clinkers, flying ashes, blast-furnace slags),

- cordierite glasses $\left(2 \mathrm{MgO}, 2 \mathrm{Al}_{2} \mathrm{O}_{3}, 5 \mathrm{SiO}_{2}\right)$ heated in pure state or in presence of raising contents of alumina, zirconia, titanium oxide, or chromium oxide. These additives allow the controlling of thermal crystallization processes.

The scanning electron microscopy is very useful for the differentiation of the observed materials versus their origin, morphology, and texture. These factors play a great role during thermal behaviour or reactivity in presence of water. In the case of cordierite glasses, the experimental technique brings interesting informations about the thermal crystallization processes, phenomenon which is influenced by the nature and contents of the oxides used as nucleation agents.
\end{abstract}

1. Introduction. - Le fait de disposer au Laboratoire d'un microscope électronique à balayage incite le chercheur à entreprendre des études morphologiques sur les matériaux dont il étudie les propriétés physico-chimiques à l'aide de techniques plus conventionnelles telles que la diffraction des rayons $\mathrm{X}$, l'analyse thermique, la magnétochimie, etc...

Dans le cadre de notre programme de recherche sur la réactivité du solide et la physicochimie des matériaux, nous étudions le comportement thermique et les réactions d'hydratation d'un certain nombrè d'aluminosilicates dont il est fait usage dans l'industrie des liants hydrauliques ou l'industrie $\mathrm{du}$ verre.

Nous allons décrire dans ce mémoire les résultats d'une étude morphologique préliminaire de clinkers de ciment portland, de cendres volantes de centrales thermiques, de laitiers de hauts fourneaux, et de verres synthétiques. Les trois premiers cités résultent d'une trempe, donc sont susceptibles de présenter un état vitrifié ou partiellement vitreux.

Les clichés présentés dans ce mémoire ont été réalisés en collaboration avec $M^{11 \mathrm{e}} \mathrm{C}$. Bardot et $M^{\text {me }} M$. Chatelut, responsables des techniques de microscopie. L'appareillage utilisé est un appareil MEB 07 CAMECA.

2. Etude des clinkers de ciment portland. - Les clinkers de ciment portland, qui donnent le ciment proprement dit après broyage, résultent de la cuisson 
à haute température, de matières premières minérales dites «cru de cimenterie » et constituées essentiellement de roches contenant de l'alumine, de la silice, de la chaux et des oxydes de fer (argiles, calcaires, marnes, etc...). La clinkérisation conduit à la formation de nodules plus ou moins sphériques, dont la dimension n'excède généralement pas le centimètre et dont la porosité interne résulte de leurs conditions de formation en phase hétérogène. Les clinkers contiennent quatre constituants minéralogiques principaux :

1. Le silicate tricalcique $3 \mathrm{CaO}, \mathrm{SiO}_{2}\left(\right.$ ou $\left.\mathrm{C}_{3} \mathrm{~S}\right)$ pseudo-hexagonal qui représente environ $55 \%$ du matériau. Le faciès de ces cristaux est généralement hexagonal et les dimensions peuvent atteindre $50 \mu$. Ce silicate contient de petites quantités d'impuretés ( $\mathrm{MgO}, \mathrm{Al}_{2} \mathrm{O}_{3}, \mathrm{Fe}_{2} \mathrm{O}_{3}, \mathrm{Cr}_{2} \mathrm{O}_{3}, \mathrm{TiO}_{2}$, etc...) en solution solide : il constitue l'alite.

2. Le silicate bicalcique $2 \mathrm{CaO}, \mathrm{SiO}_{2}$ (ou $\mathrm{C}_{2} \mathrm{~S}$ ) monoclinique. Les cristaux sont généralement de forme arrondie, parfois polygonale, mais leur aspect peut être très variable selon les conditions de cuisson et la composition du cru. Ils constituent environ $20 \%$ du clinker. De même que le silicate tricalcique, il forme des solutions solides avec de petites quantités d'impuretés et est alors dénommé bélite.

3. L'aluminate tricalcique $3 \mathrm{CaO}, \mathrm{Al}_{2} \mathrm{O}_{3}$ (ou $\left.\mathrm{C}_{3} \mathrm{~A}\right)$ cubique. Les cristaux sont de forme carrée ou rectangulaire.

4. L'aluminoferrite de calcium $4 \mathrm{CaO}, \mathrm{Al}_{2} \mathrm{O}_{3}$, $\mathrm{Fe}_{2} \mathrm{O}_{3}$ (ou $\mathrm{C}_{4} \mathrm{AF}$ ) orthorhombique.

Les deux derniers constituants représentent respectivement 12 et $8 \%$ du poids du clinker. Les cristaux d'aluminate sont bien formés si le refroidissement de clinker est lent, mais dans le cas d'un clinker trempé, l'aluminate et la phase ferrite apparaissent finement enchevêtrés. Ils constituent ce que l'on appelle la « phase interstitielle » du clinker. Selon Terrier et Hornain [1] la séparation de l'aluminate et de la ferrite peut même être bloquée par une trempe énergique au cours de laquelle la solidification leur confère l'état vitreux.

A côté de ces quatre constituants essentiels, on peut rencontrer dans le clinker des composés mineurs tels que le périclase $\mathrm{MgO}$ sous forme de petits grains anguleux, et de la chaux libre $\mathrm{CaO}$ qui apparaît généralement sous la forme de petits grains sphériques séparés ou en amas.

La méthode d'analyse minéralogique des clinkers la plus élaborée est sans conteste la microscopie optique dont l'utilisation a fait l'objet d'études très détaillées par Terrier et Coll. [1], [2] au CERILH (examens en lumière réfléchie sur sections polies, examens par transparence sur lames minces). L'observation de constituants optiquement distincts permet de distinguer les particules cristallisées et les particules vitreuses dans un mélange. L'attaque sélective par des réactifs appropriés de l'un des deux silicates du clinker permet de juger de l'homogénéité des grains laquelle dépend de nombreux facteurs (broyage et procédé de cuisson du cru, chutes de cendres dans le four, ségrégation; agglomération de poussières).

Le but que nous nous étions fixé en étudiant quelques clinkers par microscopie électronique à balayage était double :
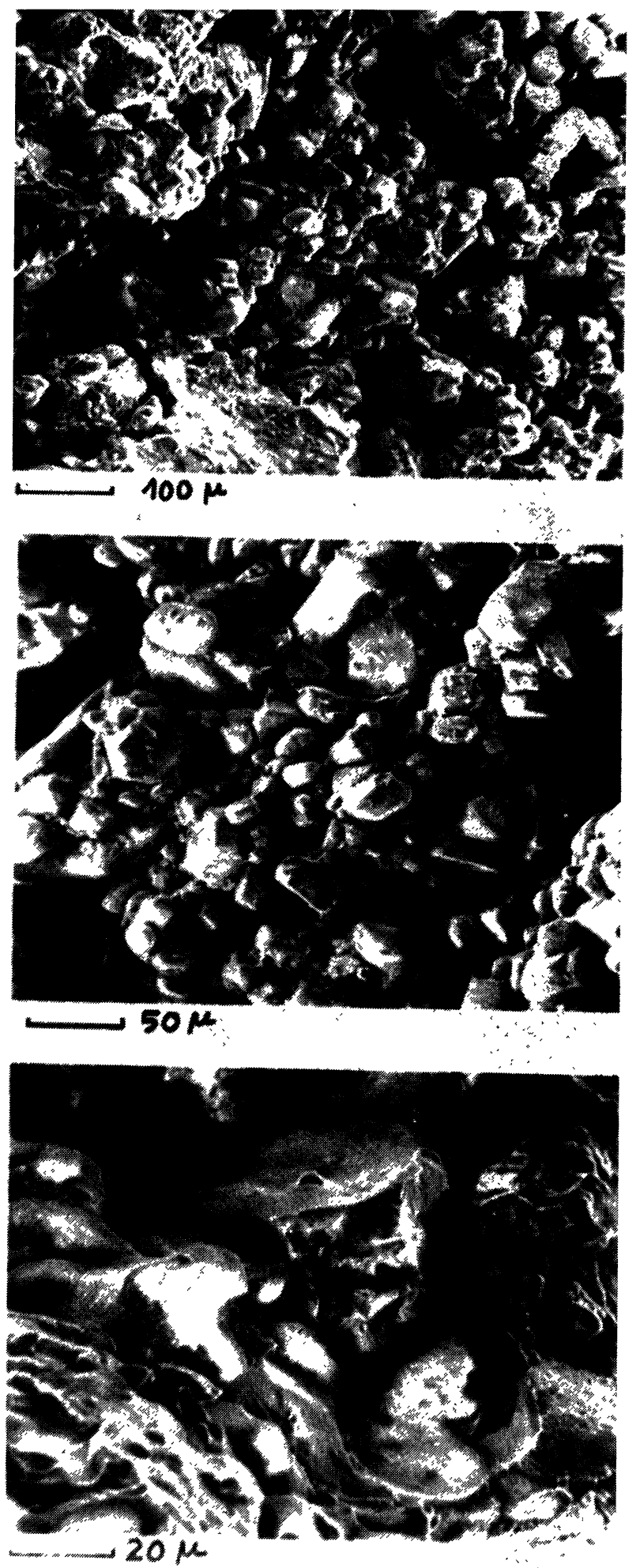

Fig. 1-2-3. - Clinker Lafarge (Usine du Val d'Azergue). 
- Observer la texture interne microscopique ce que ne permet pas la microscopie optique limitée par la profondeur de champ.

- Essayer de mettre en évidence les zones vitreuses en se basant sur des critères morphologiques.

Nous avons étudié cinq clinkers d'origines différentes. Ces matériaux nous ont été aimablement fournis par M. M. Venuat, Chef du Service Béton au CERILH. Les observations ont été faites sur fractures.

On distingue deux types très caractéristiques de texture :

- Assemblages de nodules aux formes géométriques évidentes bien que les arêtes des cristaux soient arrondies (Fig. 1 à 3 ). Il est cependant difficile de décider de la nature de ces cristaux : silicate tricalcique ou silicate bicalcique, bien que l'on discerne nettement dans quelques cas des cristaux hexagonaux de $\mathrm{C}_{3} \mathrm{~S}$ (Fig. 2). L'observation de composés purs $\mathrm{C}_{3} \mathrm{~S}$ et $\mathrm{C}_{2} \mathrm{~S}$ (respectivement Fig. 4 et 5 et Fig. 6 et 7 ) laisse à penser qu'il est difficile de prévoir le faciès de ces solides, résultants d'une synthèse en milieu probablement fondu, par observation à trois dimensions. Il est évident que la différentiation est plus
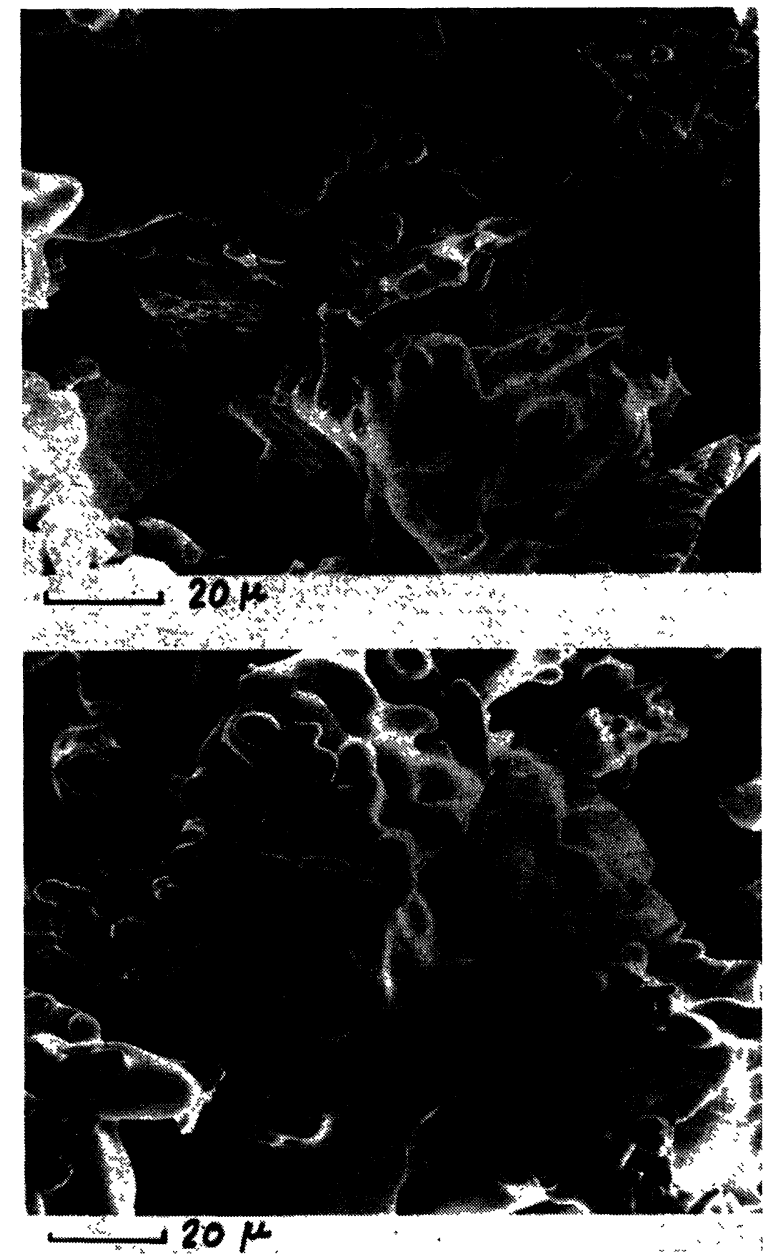

FIG. 4-5. - Silicate tricalcique synthétique Lafarge.
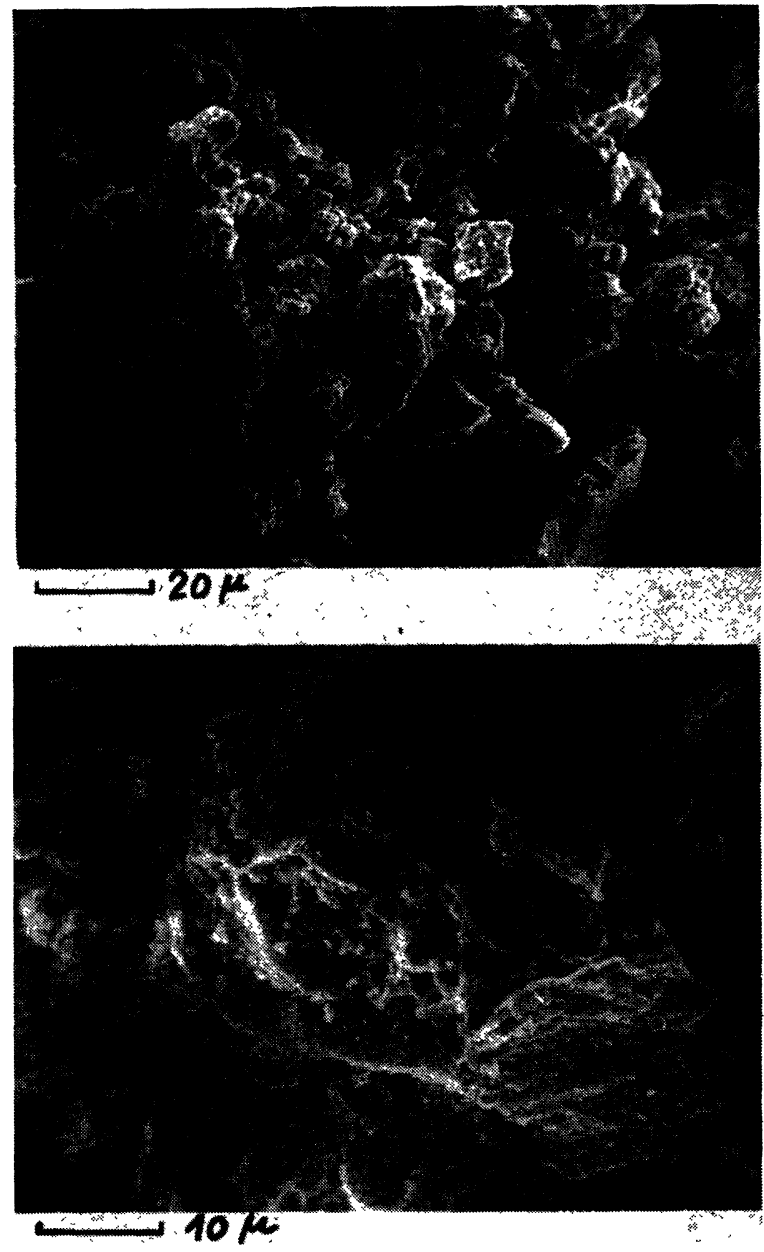

FIG. 6-7. - Silicate bicalcique synthétique Lafarge.

aisée lorsque l'on utilise la microscopie optique après polissage et attaque par des réactifs appropriés.

- Masses très tourmentées avec nombreux vides de dimensions importantes: les cristaux de silicate semblent être recouverts d'une gangue vitrifiée qui pourrait bien être la phase intersti ${ }^{+}$ielle $\left(\mathrm{C}_{3} \mathrm{~A}+\mathrm{C}_{4} \mathrm{AF}\right)$ plus ou moins vitreuse (Fig. 8 à 13). Quelques gros nodules arrondis semblent émerger de ces masses mais là encore il est délicat de décider de leur nature minéralogique.

Un échantillon présente une texture intermédiaire (Fig. 14 et 15). Les masses sont moins tourmentées (Fig. 14) et l'émergence des cristaux est plus marquée (Fig. 15).

Il n'en reste pas moins vrai que la technique permet de se faire une idée assez nette de la texture interne d'un clinker, caractéristique qui est fonction de la composition chimique du cru de cimenterie et du processus de cuisson. Mais il est difficile de se prononcer sur la phase vitreuse : en effet, les figures 4 et 5 relatives à $\mathrm{C}_{3} \mathrm{~S}$ synthétique laissent supposer que ce solide est vitrifié alors que son diagramme de diffraction met en évidence un parfait état de cristallisation. 

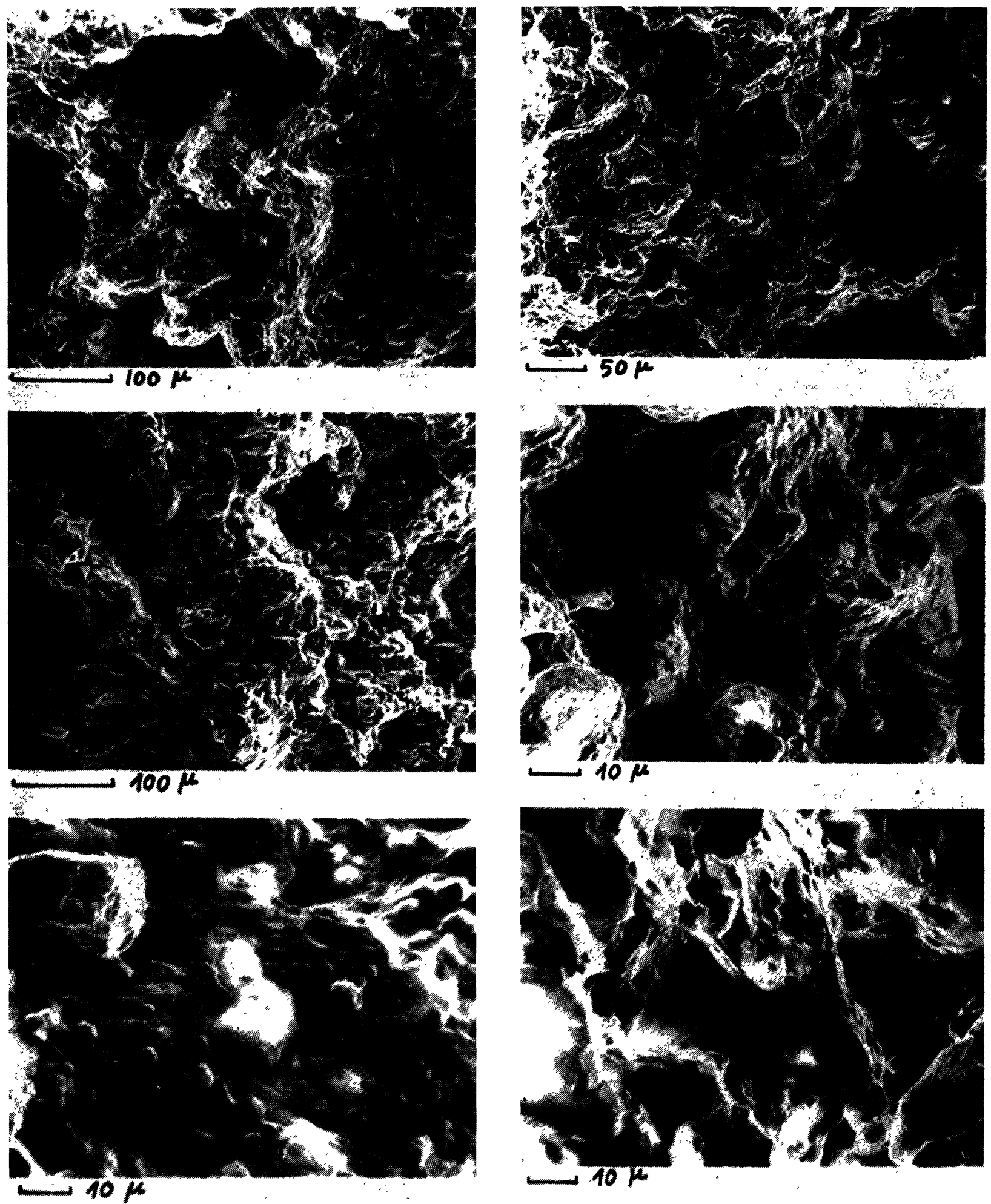

FIG. 8-9-10. - Clinker Poliet (Usine de Gargenville).

3. Etude de cendres volantes de centrales thermiques. - Les cendres volantes sont les résidus de combustion de charbon pulvérisé utilisé dans les centrales thermiques [3]. Ces matériaux sont véhiculés par les fumées et sont recueillis soit à l'aide de dépoussiéreurs électrostatiques ou collecteurs mécaniques, soit par voie humide. Le fait qu'elles aient été portées à haute

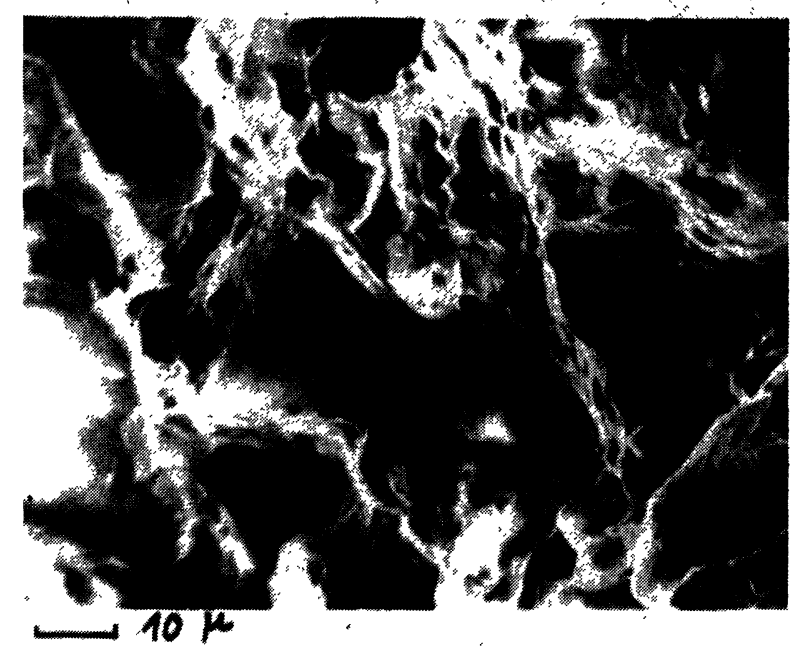

Fig. 11-12-13. - Clinker Lafarge (Usine du Teil).

température $\left(1^{\circ} 400^{\circ} \mathrm{C}\right)$ puis trempées, leur confère l'état vitreux.

Les cendres se présentent sous la forme d'une poudre constituée essentiellement de fines particules sphériques dont les dimensions sont comprises entre 0,5 et $200 \mu$. En fait la forme de grains de cendres est assez variable : une étude par microscopie optique effectuée au CERILH et rapportée par Jarrige [3] 
montre que les grains de cendres présentent souvent des formes et des couleurs variées : on discerne des billes pleines ou creuses, plus ou moins sphériques, des grains anguleux qui présentent des boursouflures et des vides (micromâchefer), des coquilles ouvertes. En général la surface des grains est lisse et brillante.

A côté de certaines cendres non typifiées et de composition variable, on distingue essentiellement les cendres silico-alumineuses (cendres de houille) et les cendres sulfocalciques (cendres de lignite).

Certaines cendres volantes sont couramment utilisées dans les bétons et les ciments portlands artificiels en raison de leur activité pouzzolanique, c'està-dire leur possibilité de libération de silice qui réagit sur la portlandite (hydroxyde de calcium) du béton durci pour donner des silicates de calcium hydratés lesquels renforcent la cohésion due à la prise des constituants hydrauliques des ciments [4].

Nos observations ont porté sur deux cendres d'origine différente :

- La cendre de la centrale de Vaires-sur-Marne (échantillon aimablement fourni par M. M. Venuat du CERILH) ;

- La cendre de la centrale thermique d'Albi. Cet échantillon nous a été fourni par MM. CarlesGibergue et Vaquier de l'INSA de Toulouse. Cette cendre a été étudiée par ces chercheurs [5] qui l'ont utilisée pour la fabrication de mortiers et qui ont mis en évidence l'action des sulfates qu'elle contient sur les processus d'hydratation.

Nous avons constaté que ces deux cendres diffèrent assez nettement par la forme, l'aspect et la dimension des grains qui la constituent (Fig. 16 et 17).

- La cendre de Vaires-sur-Marne est constituée essentiellement de sphères dont les dimensions sont comprises entre 2 et $60-70 \mu$, les plus petites formant des amas. Ces sphères présentent généralement un état de surface assez lisse (Fig. 18 et 19);

- La cendre d'Albi présente une granulométrie moyenne supérieure, les grains variant autour de 60 à $80 \mu$. On observe différents types de grains :

- Quelques sphères à surface lisse (Fig. 21) ou à surface rugueuse percées de petites cavités (Fig. 20). Certaines sphères sont ouvertes et remplies de sphères plus petites (Fig. 20).

- Des grains constitués d'un assemblage de particules de formes très variables où l'on retrouve cependant des contours sphériques lisses (Fig. 22 et 23) à côté de particules sans forme définie.

- Des grains poreux ayant l'aspect d'une véritable ponce (Fig. 24) et dont la surface est très tourmentée (Fig. 25).

Là encore l'origine différente des deux matériaux se manifeste par des morphologies très caractéristiques. Une étude en cours sur le processus d'hydratation de ces cendres doit permettre d'établir des corrélations entre ces morphologies et la réactivité de ces cendres.
4. Etude des laitiers de hauts fourneaux. - Les laitiers de hauts fourneaux sont des silico-aluminates de calcium, sous-produits de la fabrication de la fonte. La fonte est obtenue en réduisant les minerais de fer par du coke métallurgique en présence de fondant (calcaires ou roches siliceuses). Ce fondant se combine à la gangue du minerai de fer de départ pour donner le laitier, lequel est recueilli à la base du haut fourneau à l'état liquide $\left(1500^{\circ} \mathrm{C}\right)$ au-dessus de la fonte, elle-même liquide mais de densité supérieure. En France, on recueille environ une tonne de laitier pour une tonne de fonte.

Le laitier peut être refroidi lentement à l'air : il se présente alors sous la forme d'une roche compacte, généralement cristallisée, dont la réduction mécanique donne le laitier concassé utilisé en construction.

Si le refroidissement est réalisé de façon brutale en présence d'eau, on obtient un sable trempé : c'est le laitier granulé également utilisé en construction et en cimenterie.

$\mathrm{Si}$ le refroidissement est réalisé par vaporisation d'eau sous pression, la roche subit une expansion qui conduit à la formation d'alvéoles : c'est le laitier expansé utilisé dans la fabrication d'éléments de construction en béton léger.

Enfin, si le refroidissement est rapide (refroidissement à l'air comprimé) après que le laitier ait été refondu à $1300^{\circ} \mathrm{C}$, il y a formation de la laine de laitier utilisable pour l'isolation phonique et thermique [4].

En général les laitiers sont vitrifiés par refroidissement rapide. Cette vitrification augmente la réactivité du matériau vis-à-vis de l'eau (moyennant la présence de catalyseurs, en l'occurrence des bases - chaux, soude - ou des silicates alcalins) et leur confère ainsi un pouvoir hydraulique, mais ces propriétés sont tributaires de la composition chimique qui doit respecter certaines limites [4].

Il apparaît donc que selon son origine et le protocole de trempe, le laitier pourra présenter une morphologie assez caractéristique.

Nos recherches sur le comportement thermique des matériaux d'une part, et sur leur hydraulicité d'autre part, nous ont conduits à entreprendre une étude morphologique préliminaire sur des laitiers de différentes origines (France, Espagne, Italie). Les diagrammes de Guinier en chambre de Nonius ne laissent aucun doute quant à l'état vitreux des échantillons que nous avons choisis : tous les spectres sont pratiquement blanes.

Le laitier français présente trois types de granulats de couleurs très nettement différentes : noire, brune et blanche. Les figures 26 et 27 montrent que les grains noirs sont constitués de nodules sans forme nettement définie, à surface lisse et à lisières anguleuses. On note quelques plages où se distinguent des particules plus petites à contour géométrique assez net (Fig. 27).

Les grains bruns (Fig. 28 et 29) sont constitués d'assemblages de nodules ovoïdes présentant des 

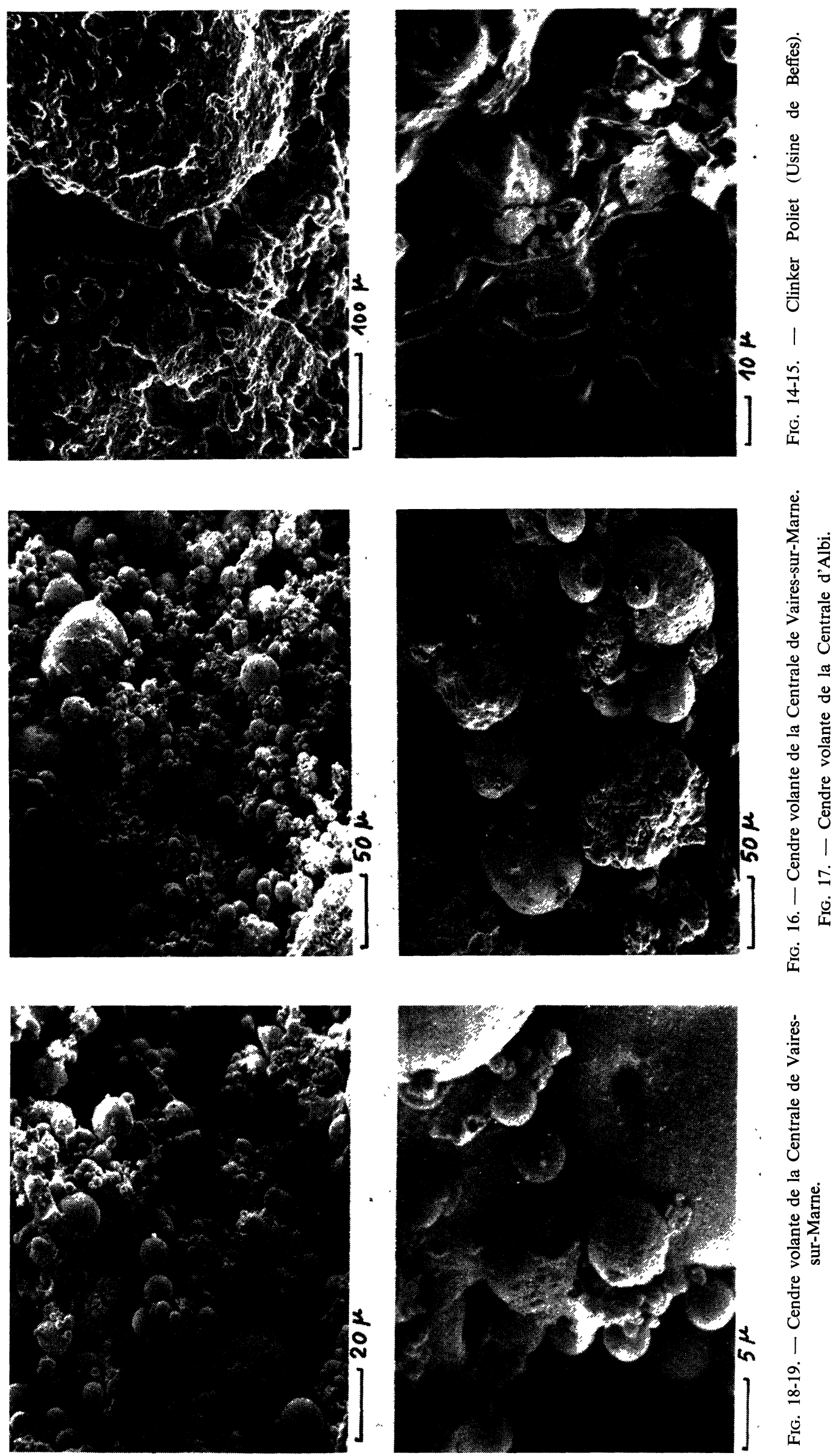

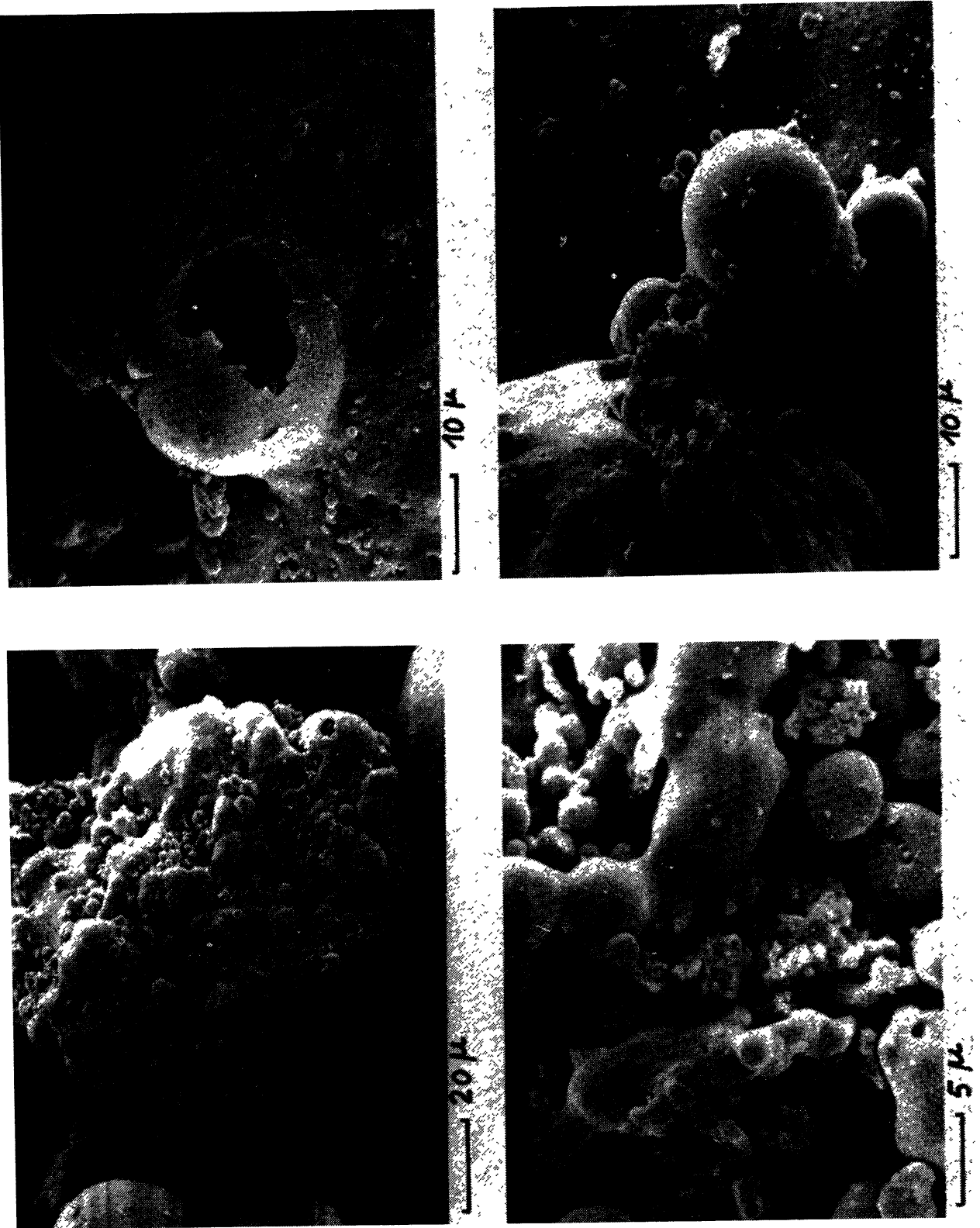

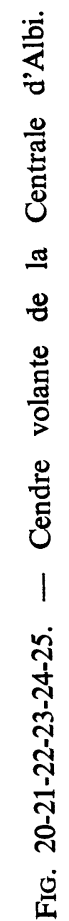
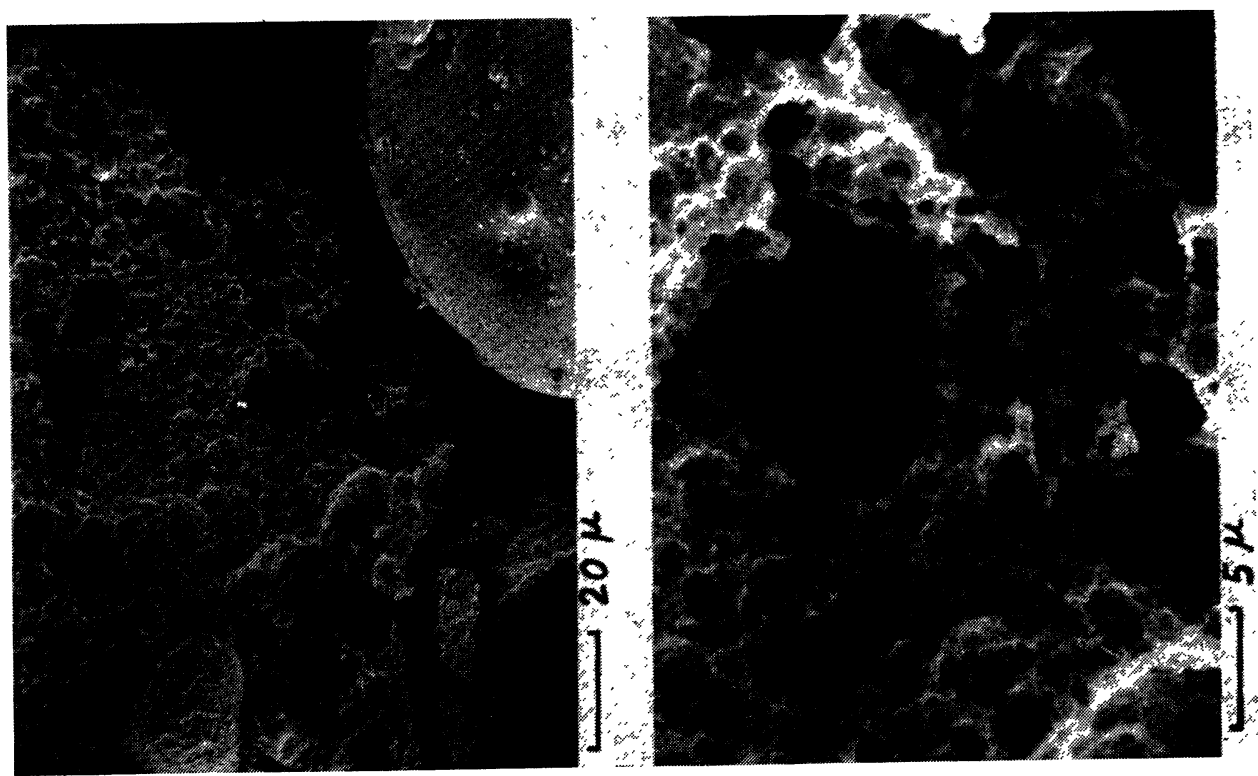

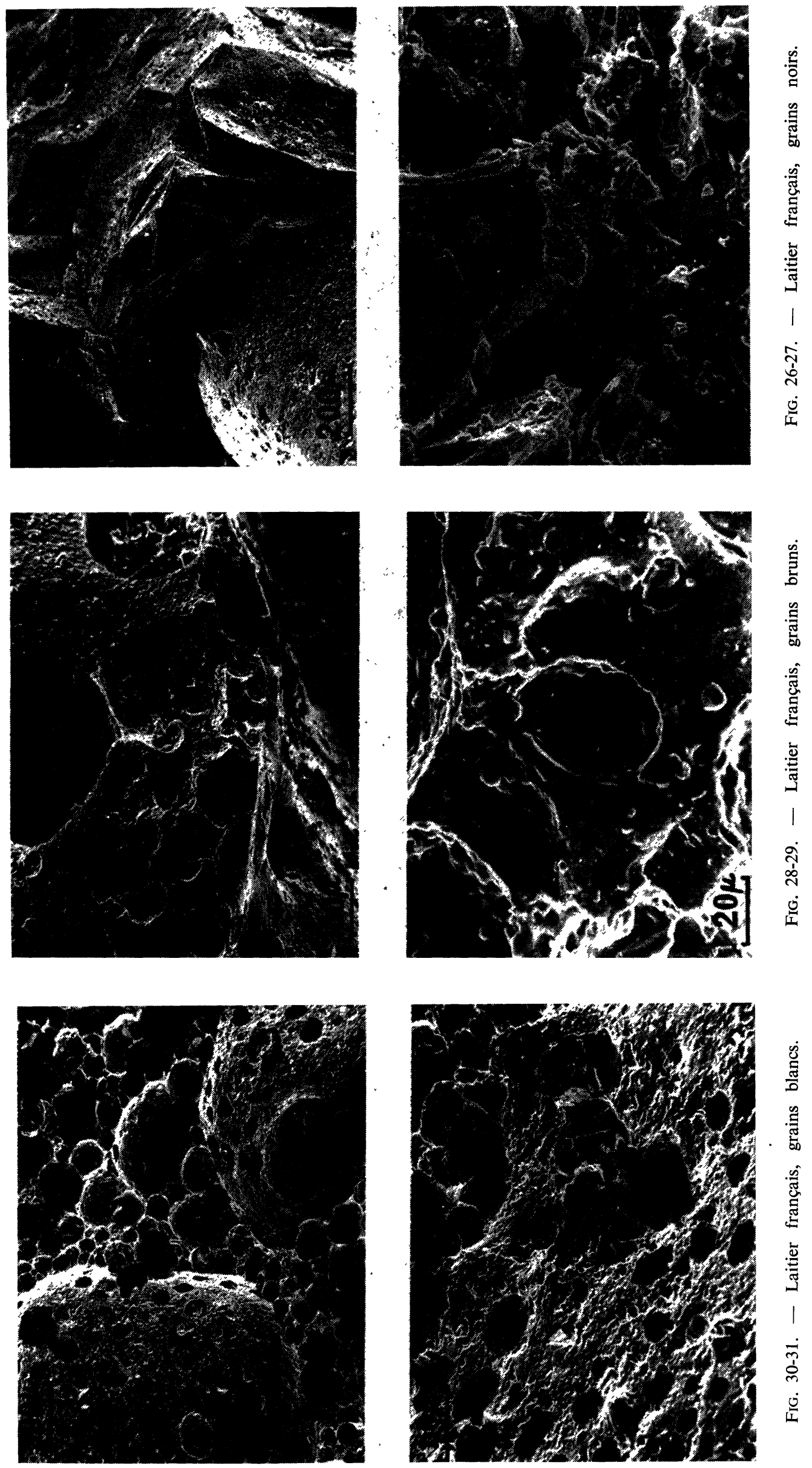
zones caverneuses et des protubérances en forme de pustules. Les grains blancs (Fig. 30 et 31 ) sont également constitués d'assemblages de nodules dont la forme est plus sphérique et dont les dimensions sont variables. La surface de ces nodules présente de nombreux cratères et cavités hémisphériques. Le fait de trouver ces trois types de grains dans le laitier tel quel de départ laisse supposer que chacun de ces types présente une composition propre en corrélation probable avec sa morphologie.

Le laitier espagnol, de couleur claire, est très différent des précédents (Fig. 32 et 33). On n'observe qu'un seul type de grains constitués de blocs caverneux à texture plus ou moins spongieuse. Ces blocs sont formés d'assemblages jointifs de nodules sphériques d'environ $200 \mu$ de diamètre, dont la structure superficielle est assez particulière et caractérisée par l'existence de nombreuses cavités de forme géométrique assez bien définie.

Les trois échantillons de laitier italien ne diffèrent pas du point du vue macroscopique : la taille des particules est sensiblement la même mais la couleur est assez différente : blanchâtre, verte et grise. Tous trois présentent un aspect assez tourmenté avec existence d'arêtes vives et de plis assez caractéristiques, et présence de nombreuses cavités superficielles,
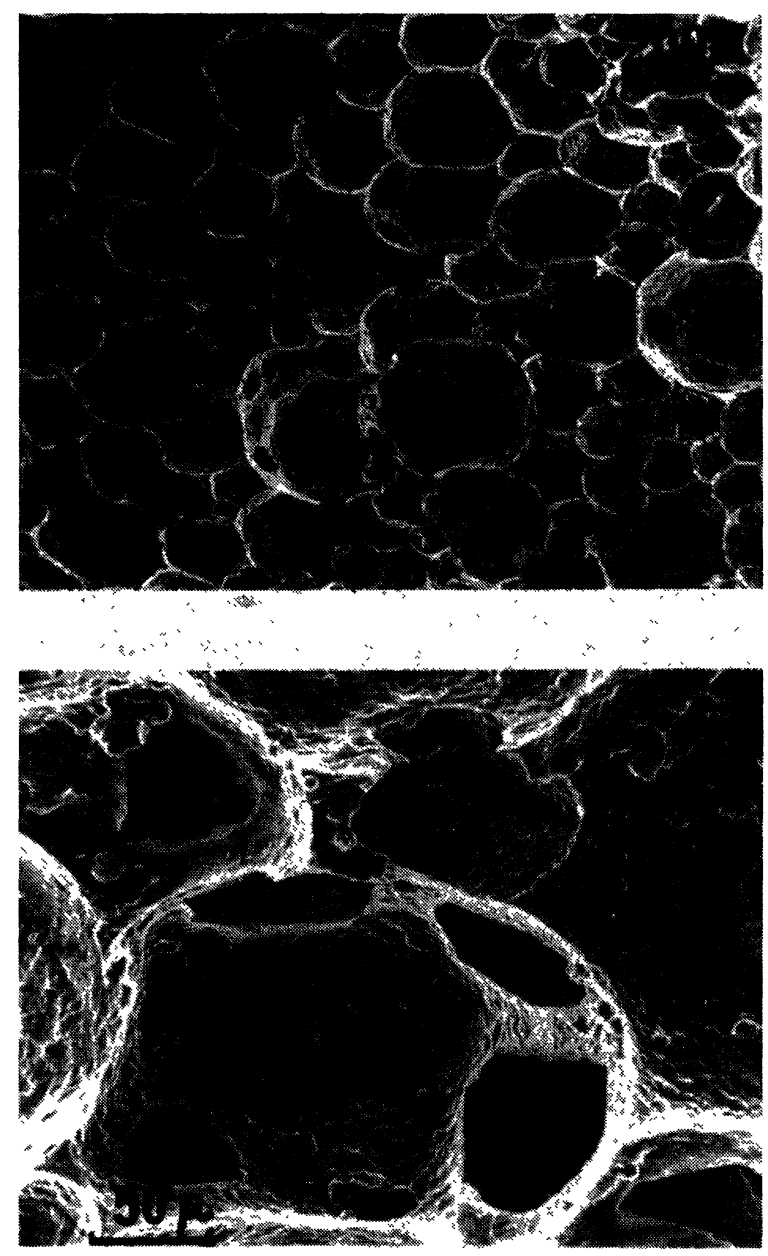

FIG. 32-33. - Laitier espagnol.

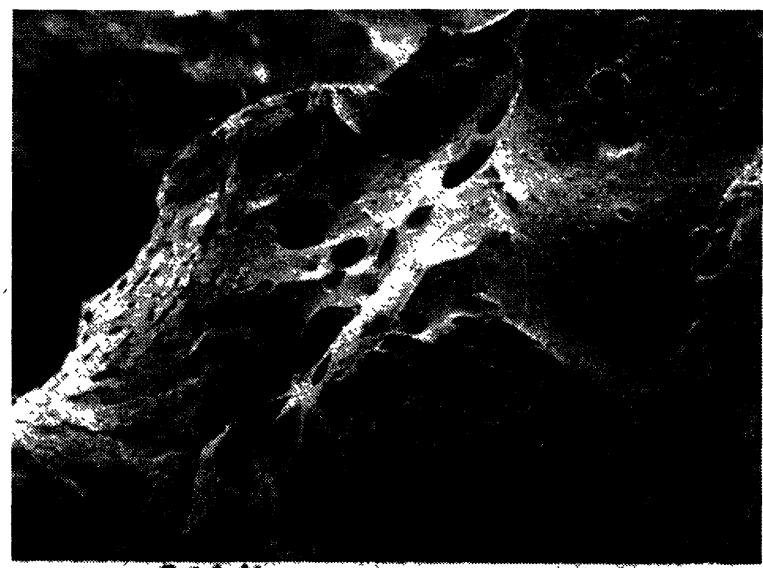

$200 \mu$

(1)

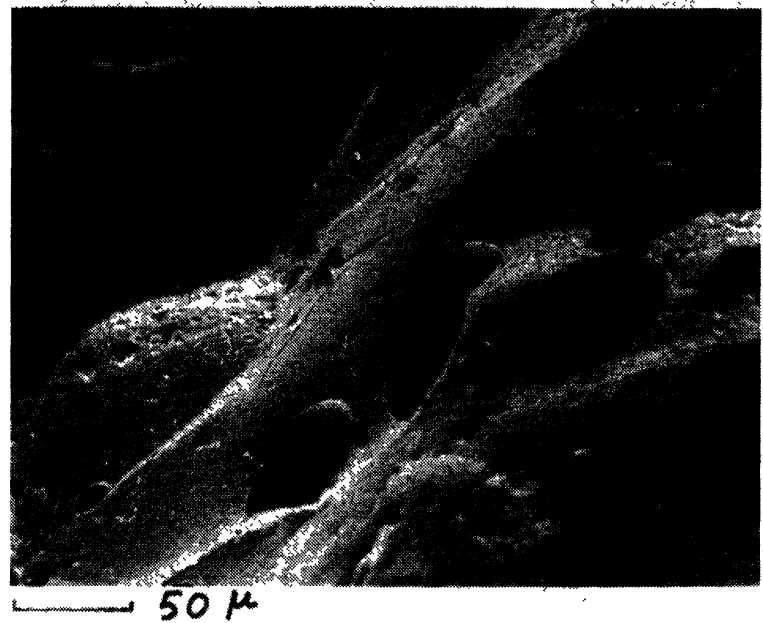

FIG. 34-35. - Laitier italien blanc.

cavités généralement à contour circulaire ou ovoïde et dont le diamètre et les dimensions sont variables pour chaque échantillon. Les plus grandes sont observées avec le laitier vert (Fig. 36 et 37) et leur contour est assez net comme pour le laitier blanc d'ailleurs (Fig. 34 et 35). Ces contours sont plus accidentés dans le cas du laitier gris (Fig. 38 et 39).

Il est certain que le processus de formation des phases hydratées résultant de la réaction de ces solides avec l'eau, doit être très variable selon la morphologie et la composition. Des études sont en cours à ce sujet mais il serait prématuré de tirer des conclusions dans l'état actuel de cette recherche. L'état de vitrification joue un rôle important et il est donc intéressant de vouloir faire un rapprochement entre la réactivité et le mode d'obtention du matériau, donc sa morphologie.

5. Etude des verres de cordiérite. - Les travaux de l'un d'entre nous [6] portent sur l'étude des phénomènes de recristallisation des verres et sur la préparation de céramiques vitreuses. La préparation de tels matériaux nécessite l'addition au verre de substances particulières (agents de germination) afin de contrôler les processus physicochimiques qui se 

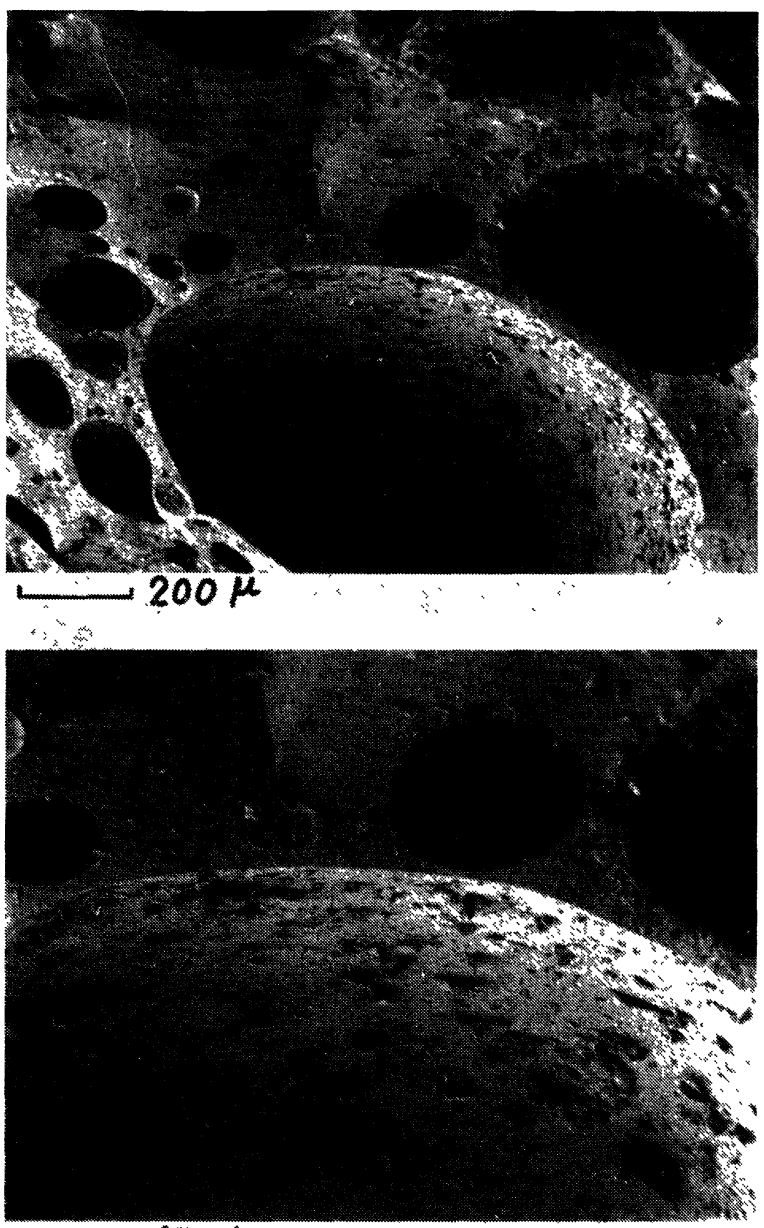

$100 \mu$

FIG. 36-37. - Laitier italien vert.

manifestent au cours de l'évolution thermique du solide et en particulier la croissance des germes. La germination peut en effet, être homogène ou hétérogène :

- homogène si elle résulte de la formation, sur les grains de verre, de régions discrètes de phase cristalline dont la composition est la même que celle du cristal qui va croître par la suite,

- hétérogène quand elle se manifeste après addition au verre - soit de phases cristallines insolubles (oxydes à action catalytique tels que $\mathrm{ZrO}_{2}, \mathrm{TiO}_{2}$, etc...) soit de suspensions colloïdales d'un métal noble jouant le rôle de germe.

La cristallisation globale n'est aisément contrôlable que si les températures respectives de germination et de croissance de germes sont suffisamment éloignées. En outre, la texture finale de la céramique est en relation très étroite avec la cinétique de germination : une germination lente conduit à un nombre limité de cristallites dont la taille est importante; par contre, une germination rapide entraîne la formation d'une céramique finement grenue comportant un nombre important de microcristallites.

L'utilisation de la microscopie électronique à
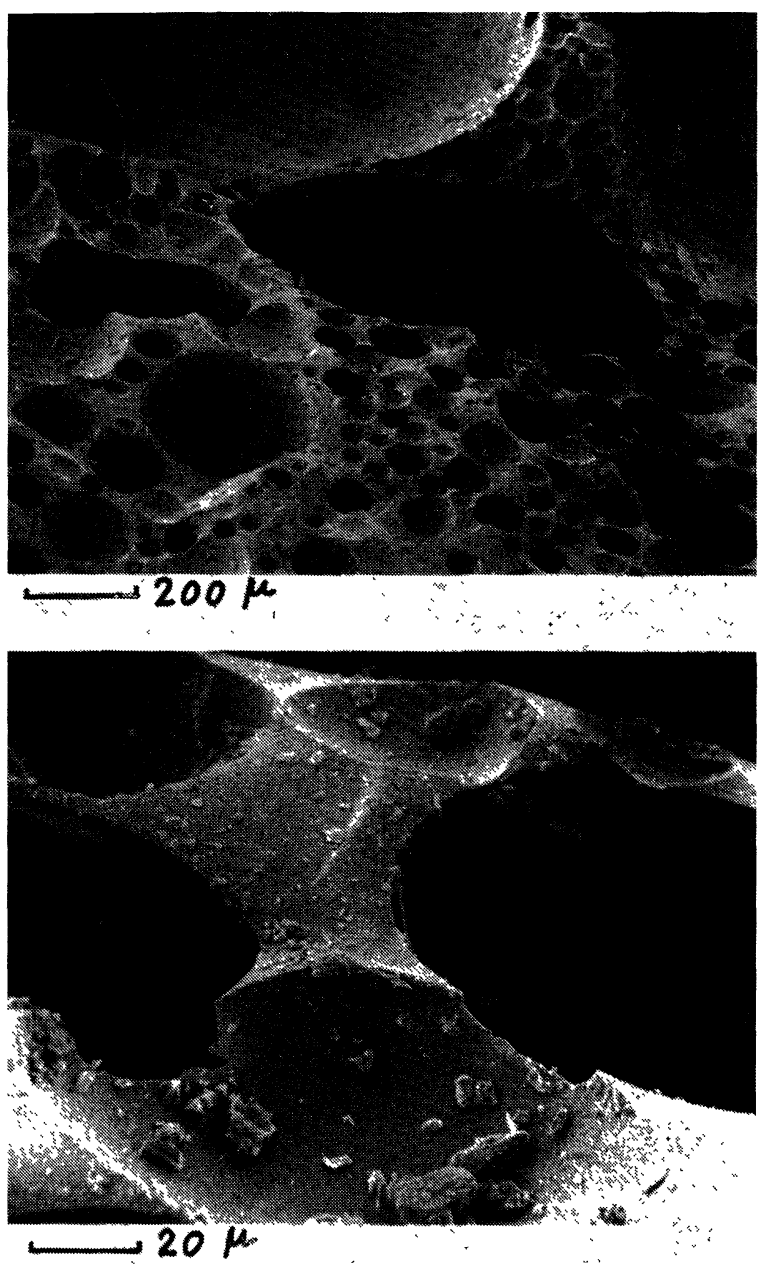

FIG. 38-39. - Laitier italien gris.

balayage est donc tout indiquée pour observer les phénomènes de recristallisation proprement dits et la texture des céramiques vitreuses.

Nos observations ont porté sur un verre de cordiérite $2 \mathrm{MgO}, 2 \mathrm{Al}_{2} \mathrm{O}_{3}, 5 \mathrm{SiO}_{2}$ pur ou additionné d'oxydes métalliques (zircone, alumine, oxyde de titane, oxyde de chrome) susceptibles de catalyser la germination.

Les résultats des études radiocristallographiques, des recherches par analyse thermique, et des observations par microscopie optique ne seront pas développés ici. Notons simplement qu'en analyse thermique différentielle, le pic de recristallisation de la cordiérite vers $1015^{\circ} \mathrm{C}$ se dédouble quand on ajoute un oxyde au verre, ce qui traduit l'apparition transitoire de cordiérite cristallisée.

5.1 Résultats Des OBSERVATIONS SUR POUDRES. La poudre de verre de cordiérite, non traitée thermiquement, est constituée de grains de dimensions variables $(5$ à $80 \mu)$ et de morphologie assez bien définie, caractérisée essentiellement par la présence d'arêtes vives (Fig. 40). A grossissement plus élevé, on constate que la surface des grains est généralement lisse (Fig. 41). 


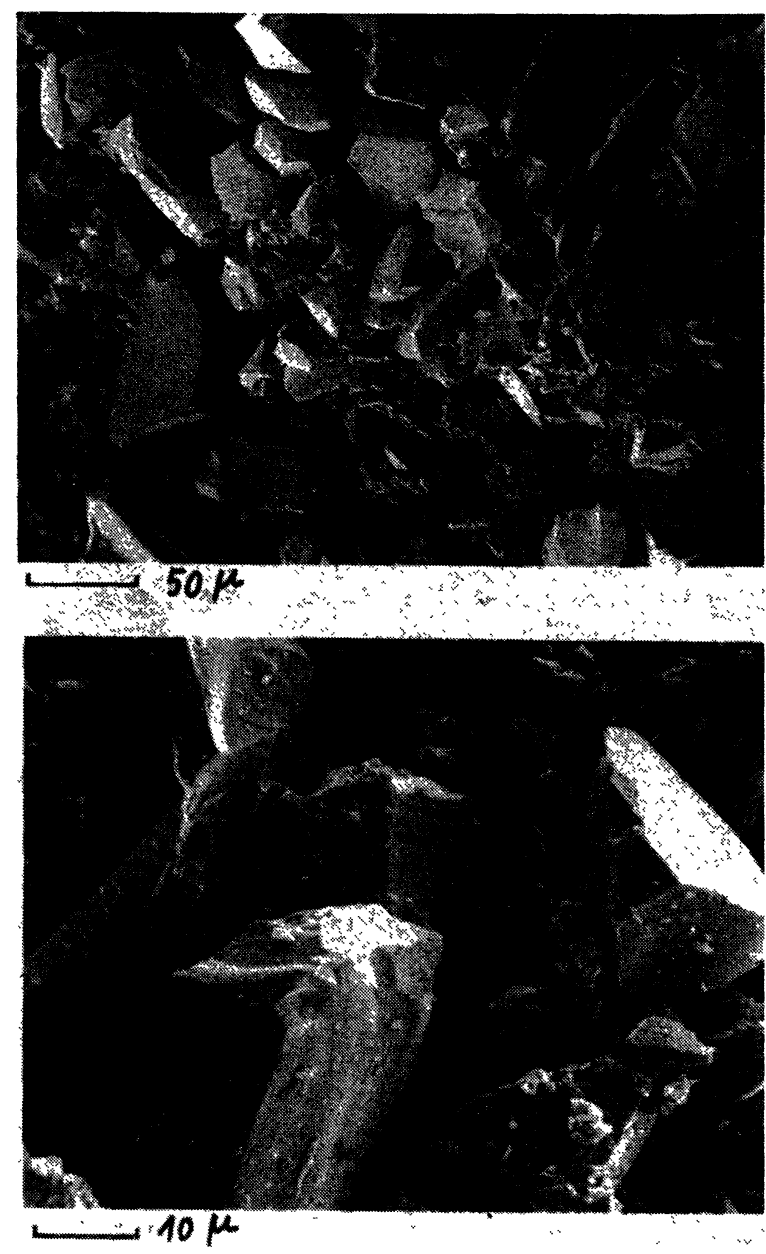

FIG. 40-41. - Verre de cordiérite pur.

La morphologie des grains de cordiérite chauffée à $1300{ }^{\circ} \mathrm{C}$ à l'air change nettement : on assiste à un arrondissement très marqué des grains avec disparition des arêtes vives (Fig. 42 et 43).

Les verres contenant des oxydes ont été étudiés après traitement thermique à une température immédiatement supérieure à celle à laquelle se manifestent les effets thermiques d'ATD, puis refroidissement. On constate généralement que les grains présentent alors des aspects d'autant plus différents de ceux du verre pur (traité dans les mêmes conditions) que la quantité d'oxyde ajouté est plus grande.

Ainsi le verre contenant $10 \%$ de zircone (Fig. 44) ne semble pas présenter de différences marquantes par rapport au verre pur, mais dès que l'addition de zircone est de $20 \%$, on assiste à l'apparition, sur les grains, de microcristallites souvent regroupés en assemblages plus ou moins compacts (Fig. 45). Avec $40 \%$ de zircone, on constate que de nombreux grains ne présentent plus une surface lisse mais prennent l'aspect d'éponges (Fig. 46 et 47) ou sont partiellement recouverts d'assemblages de microcristallites dont la taille n'excède pas le micron. Il en est sensiblement de même pour une addition
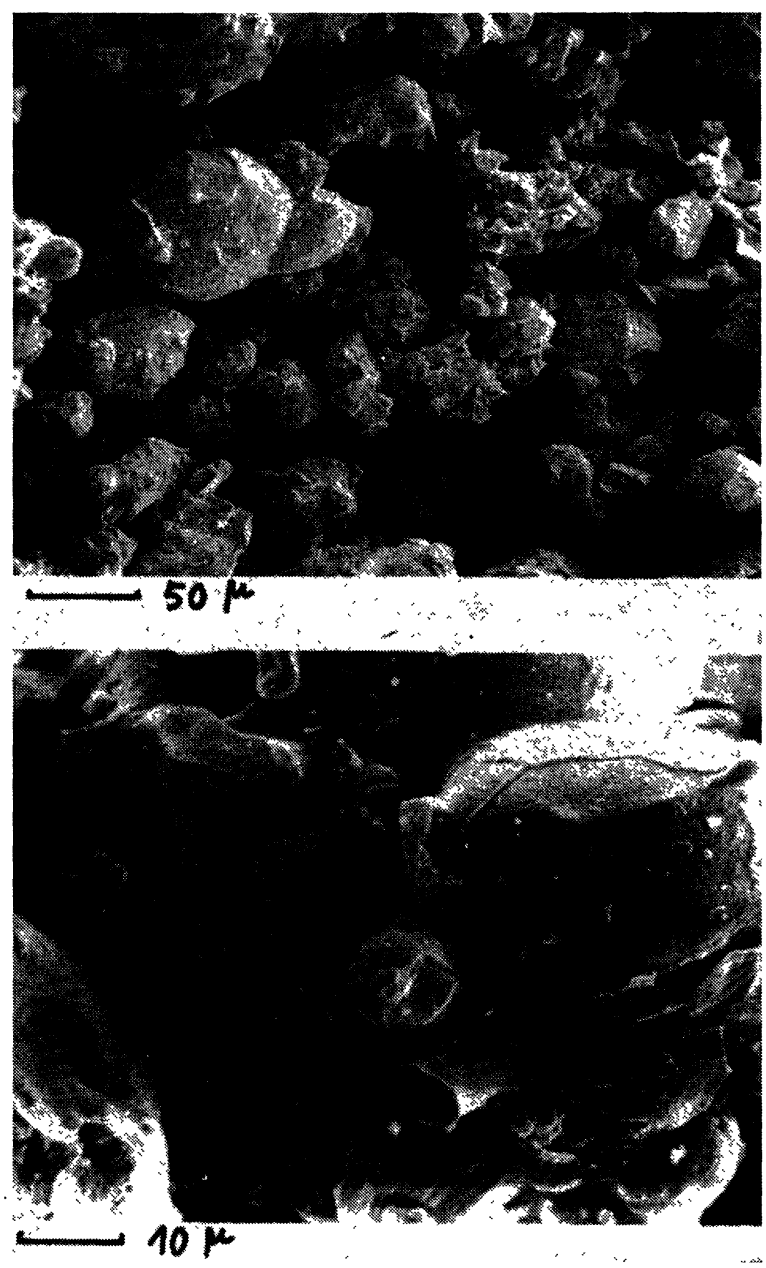

Fig. 42-43. - Verre de cordiérite pur chauffé à l'air à $1300^{\circ} \mathrm{C}$.

de zircone de $60 \%$ (Fig. 48 et 49). Avec $90 \%$ de zircone, pratiquement tous les grains sont spongieux (Fig. 50 et 51). A grossissement plus élevé (8 000) on constate que ces cristallites sont de forme sphérique (Fig. 52 et 53 ).

Les observations sont sensiblement identiques, que l'on ait observé un solide traité juste au-delà du premier pic d'ATD ou immédiatement après la manifestation du second pic.

Cette cristallisation, qui apparaît d'autant plus marquée que la teneur en oxyde étranger est plus grande, varie avec la nature de l'oxyde utilisé. Ainsi, les cristaux qui apparaissent à la surface des grains de verre dans le cas de l'addition d'oxyde de titane (Fig. 54-55) ou d'oxyde de chrome (Fig. 56-57) sont de dimensions légèrement inférieures à celles des verres contenant la zircone. Par contre, dans le cas d'une addition d'alumine, les cristallites sont de dimensions beaucoup plus élevées (Fig. 58-59) et présentent la forme de haricots.

5.2 RÉSUltats D'OBSERVATIONS SUR CÉRAMIQUES VITREUSES. - Ces céramiques sont obtenues par traitement thermique à l'air (ou sous hydrogène) de poudres de verre préalablement compactées, et auxquelles des 

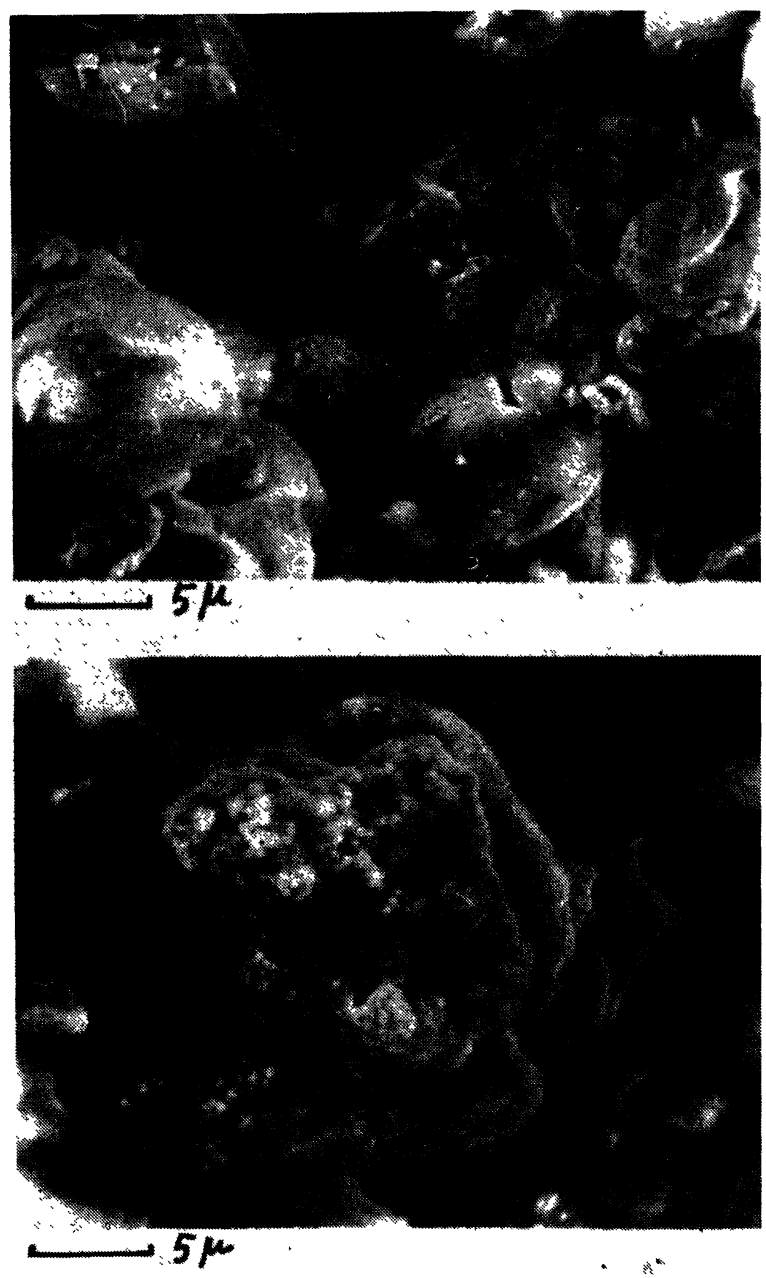

FIG. 44. - Verre de cordiérite contenart $10 \%$ de zircone, traité thermiquement.

Fig. 45. - Verre de cordiérite contenant $20 \%$ de zircone, traité thermiquement.

quantités déterminées d'oxydes métalliques ont été préalablement ajoutées.

L'observation, par microscopie optique, de la surface des échantillons permet de se faire une idée assez précise de la dimension et de la distribution des constituants du matériau composite.

L'observation, par microscopie électronique à balayage sur fracture, apporte des renseignements sur la texture interne de ces céramiques. Il est cependant nécessaire, dans ce cas, d'être prudent dans les interprétations, une fracture étant souvent une zone fragile du matériau, donc son observation n'est pas forcément représentative de la texture interne globale.

Nous donnons à titre d'exemple les différences assez importantes que l'on peut mettre en évidence, en fonction de la nature de l'oxyde initialement ajouté $(90 \%)$ dans le cas de céramiques de verre de cordiérite contenant de l'oxyde de chrome (Fig. 60), de l'oxyde de titane (Fig. 61), de l'alumine (Fig. 62) ou de la zircone (Fig. 63). La texture de la céramique contenant de l'oxyde de chrome est très serrée et résulte d'un
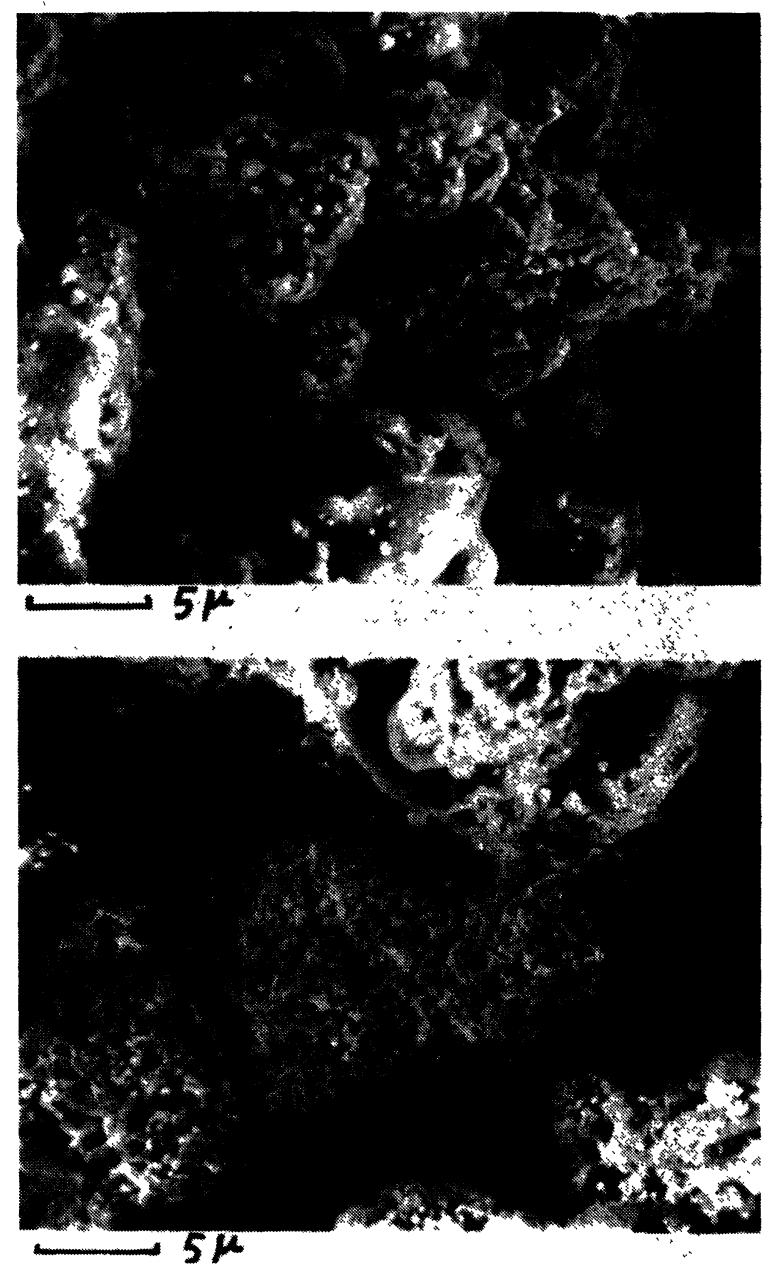

Fig. 46-47. - Verre de cordiérite contenant $40 \%$ de zircone, traité thermiquement.

enchevêtrement de microcristallites. Elle est probablement plus poreuse que celle des trois autres échantillons lesquels présentent des amas massifs et des cavités formées probablement pendant le frittage thermique. Ces cavités sont d'ailleurs tapissées de grains de forme différente selon la nature de l'oxyde ajouté : grains polyédriques dans le cas de l'alumine (Fig. 64) et grains sphériques, très petits, dans le cas d'addition de zircone (Fig. 65).

Une ét '’de en cours doit permettre de voir l'influence de la quantité d'oxyde ajoutée sur la texture de ces matériaux.

6. Conclusion. - Nous avons présenté quelques exemples d'observation de matériaux vitreux par microscopie électronique à balayage. Ces études préliminaires étaient nécessaires compte tenu des recherches menées parallèlement et relatives à l'étude de la réactivité de ces solides. Nous avons pu constater que la texture et l'aspect de ces matériaux différaient grandement de ceux généralement observés par microscopie optique. Le cas est particulièrement frappant pour les clinkers de ciment portland et les laitiers. On retrouve 

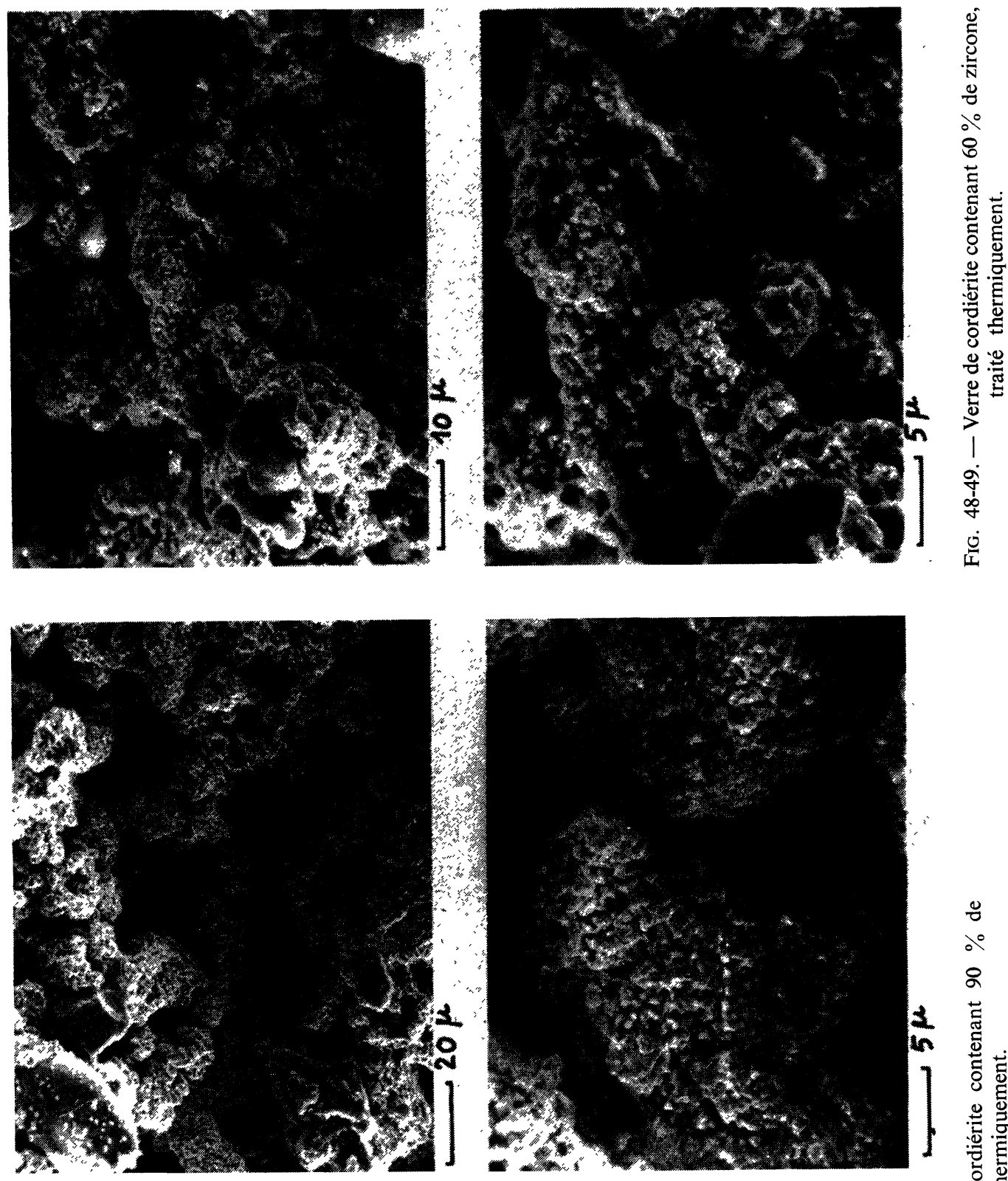

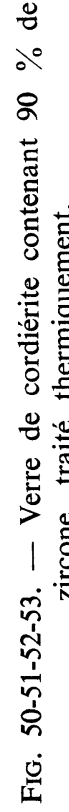
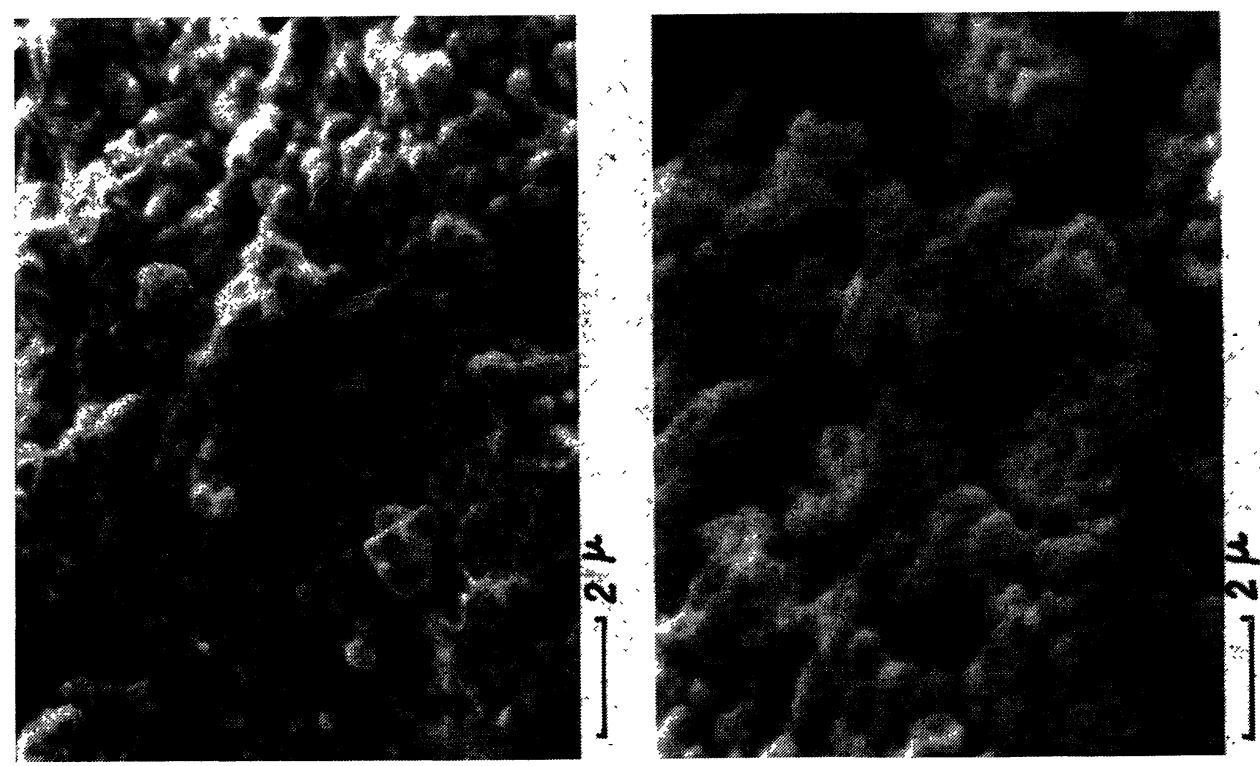

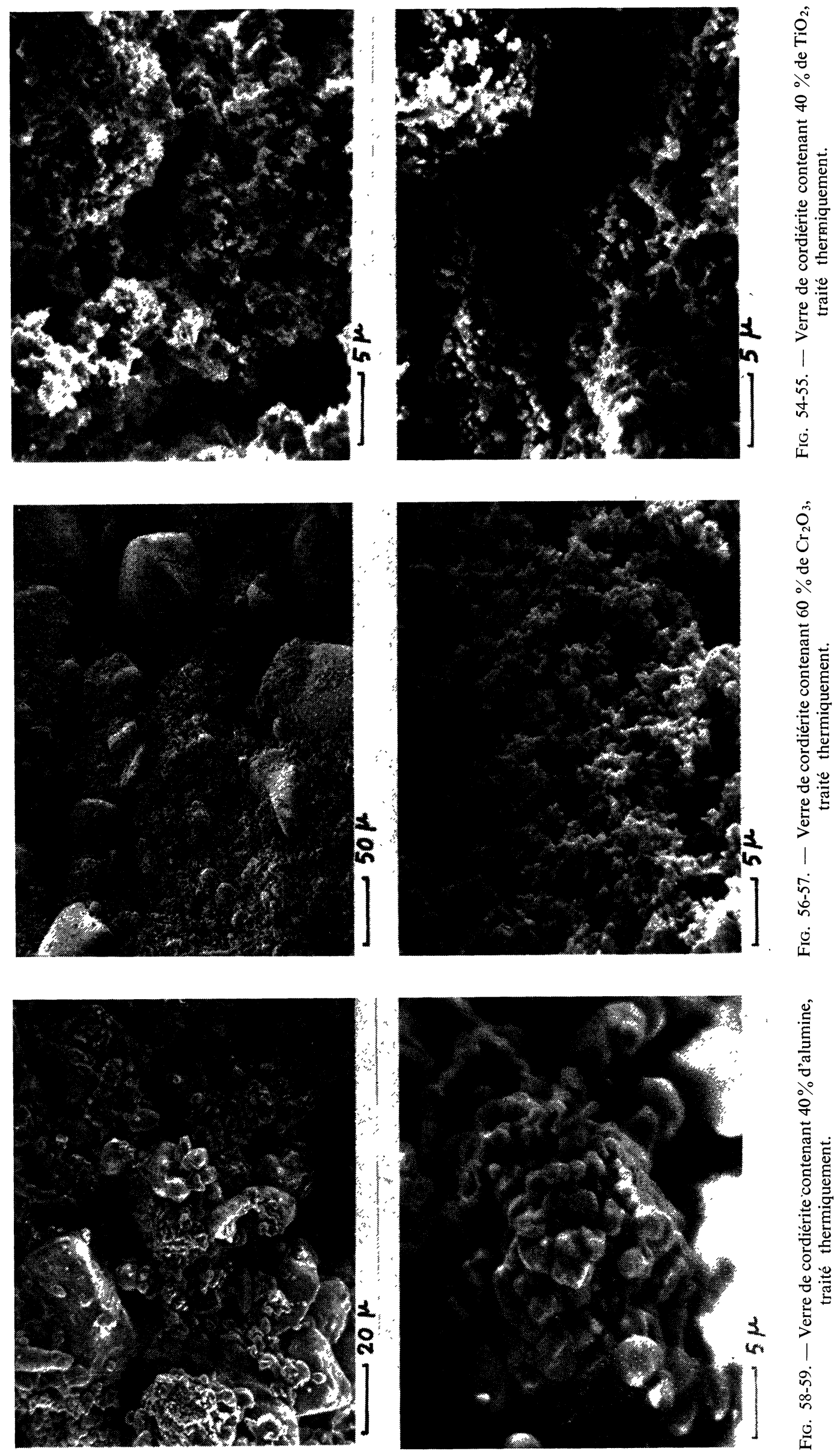

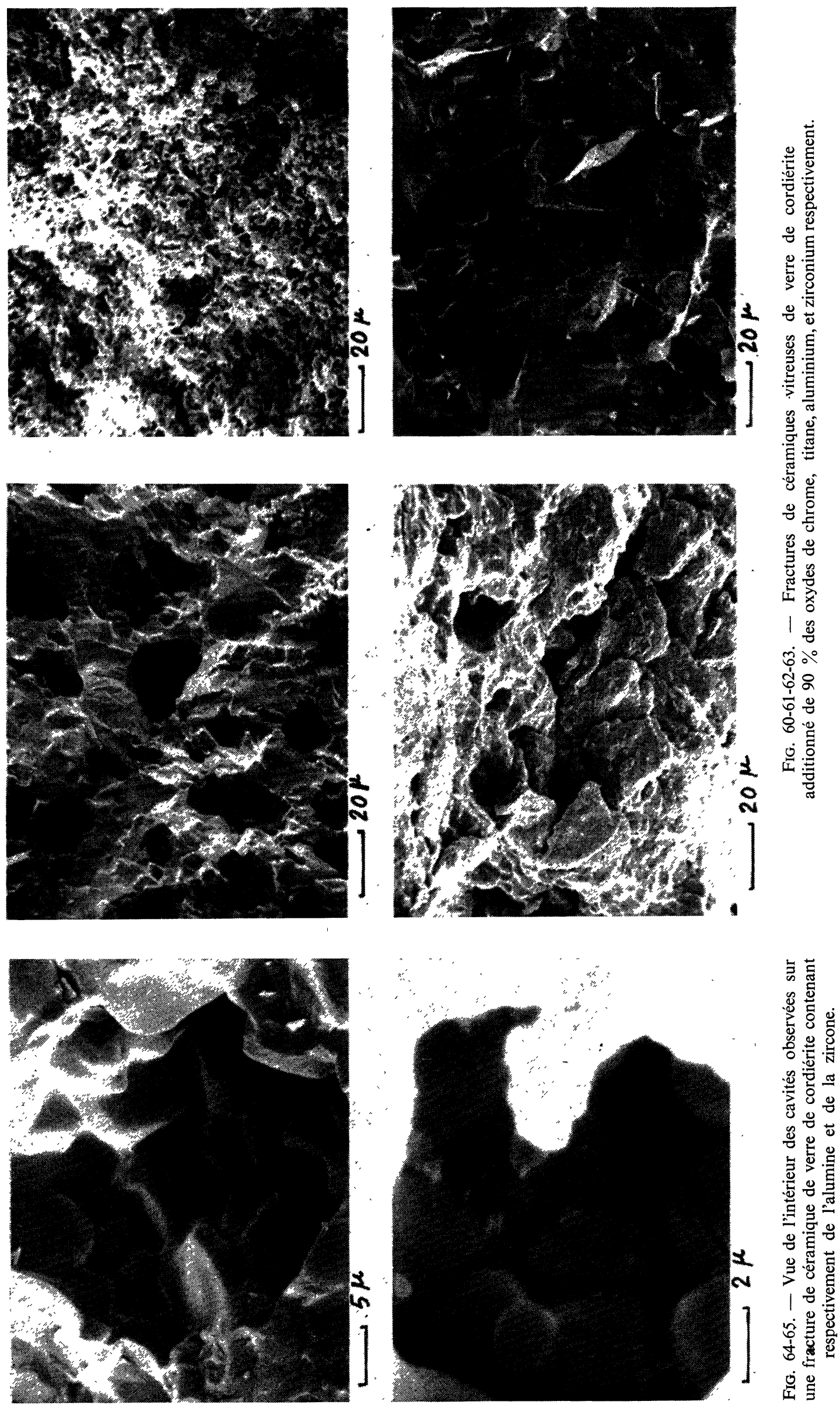
pour les cendres volantes de centrales thermiques les grains sphériques qui les caractérisent, mais la microscopie électronique à balayage permet de déceler des particularités caractéristiques de chaque cendre. Enfin, la technique expérimentale utilisée permet de suivre la recristallisation des verres et d'observer, sur fractures, la texture interne des céramiques vitreuses, texture qui dépend de la nature de l'oxyde préalablement ajouté au verre aux fins de contrôle des phénomènes de recristallisation.

\section{Bibliographie}

[1] Terrier, P., Hornain, H., Rev. Mat. Constr. no 618 à 621, (1967).

[2] Allegre, R., Terrier, P., Rev. Mat. Constr., no 501 (1957);

Minerbe, M., Terrier, P., Rev. Mat. Constr., n ${ }^{0} 508$ (1958).

[3] Jarrige, A., " Les Cendres Volantes » Eyrolles, Paris, 1971.
[4] Papadakis, M., Venuat, M., Fabrication et Utilisation des Liants Hydrauliques, Paris (23, rue de Cronstadt), 1966 ( $2^{\mathrm{e}}$ éd.).

[5] Carles-Gibergues, A., Stambolieva, R., Vaquier, V., Mat. et Constr. 6 (1973) 29-38.

[6] Amato, I., Negro, A., Ceramurgia (Ital.) 1 (1971) 16-28. 